\title{
PREMESSA ALLA PRIMA EDIZIONE (2006)
}

Con notevole ritardo rispetto al previsto, dovuto ad inopinate difficoltà di vario genere, vede oggi finalmente la luce il primo volume del nostro Nomenclator metricus Graecus et Latinus, che ripete il titolo, ma non certo l'agilità, l'originalità e la genialità del Nomenclator metricus pubblicato a Heidelberg nel 1929 da Otto Schroeder. Si tratta, infatti, molto piú modestamente di un indice sistematico dei termini tecnici impiegati in ambito metrico dalle fonti antiche, greche e latine, illustrati attraverso le testimonianze superstiti.

L'opera è organizzata per voci, redatta dai collaboratori elencati in calce al volume. Le voci sono ordinate alfabeticamente, ma ripartite per ovvie esigenze di carattere alfabetico in due grandi sezioni: nella prima sono contemplati i termini greci, unitamente - se del caso - ai loro eventuali equivalenti latini; nella seconda figureranno invece i termini peculiari della tradizione latina, mentre opportuni rinvii provvederanno ai raccordi.

Sotto ogni voce, poi, sono riportate in successione cronologica le testimonianze relative, suddivise anch'esse, all'occorrenza, in due sezioni: la prima riservata agli autori greci, la seconda ai latini. Quando però l'eterogeneità e la complessità del materiale raccolto sotto lo stesso lemma lo richiedono, non si manca di procedere ad ulteriori e piú articolate suddivisioni, inaugurate talora per maggiore chiarezza da didascalie o da schemi metrici.

Per gli autori censiti ci si è attenuti alle migliori e piú recenti edizioni, segnalando peraltro gli sporadici interventi testuali giudicati necessari dai vari collaboratori. Quanto al taglio e alla scelta delle testimonianze, si è preferito non di rado largheggiare, in modo da offrire per ciascun termine contesti che ne rispecchiassero adeguatamente il valore documentario e fornissero insieme la misura della sua vitalità nello spazio e nel tempo.

Inutile precisare che l'opera non presume affatto di essere esaustiva, né per quanto riguarda i lemmi considerati, né per quanto riguarda il materiale registrato sotto ciascun lemma. Anzi, è appena il caso di avvertire che per molti termini, specie per quelli piú comuni e di plurisecolare consumo, si è ritenuto di fornire soltanto un'ampia campionatura, privilegiando ovviamente le attestazioni piú antiche e le testimonianze delle fonti di piú spiccata caratura tecnica.

Mi corre infine l'obbligo di ringraziare vivamente i componenti dei gruppi di ricerca sulla tradizione grammaticale latina operanti presso le Università di Roma Tre, di Padova, di Trieste e di Napoli 'L'Orientale', coordinati da Mario De Nonno, per la collaborazione scientifica e il sostegno economico che hanno consentito la realizzazione dell'opera.

Un ringraziamento particolare va inoltre al Dipartimento di Scienze dell'Antichità dell'Università di Padova, che fin dall'inizio ha ospitato e favorito il lavoro redazionale.

Giuseppe Morelli 